\title{
Hookwire-guided Sentinel Lymph Node Biopsy Using Contrast-enhanced Ultrasonography Followed by a One-step Nucleic Acid Amplification (OSNA) Assay for Breast Cancer
}

\author{
TOMOHIRO MIYAKE, KENZO SHIMAZU, TOMONORI TANEI, YASUTO NAOI, \\ NAOFUMI KAGARA, MASAFUMI SHIMODA, SEUNG JIN KIM and SHINZABURO NOGUCHI
}

Department of Breast and Endocrine Surgery, Osaka University Graduate School of Medicine, Osaka, Japan

\begin{abstract}
Aim: To investigate the feasibility of hookwireguided sentinel lymph node biopsy (SLNB) using contrastenhanced ultrasonography (CEUS) followed by a one-step nucleic acid amplification (OSNA) assay. Patients and Methods: Clinical T1-2NOMO breast cancer patients scheduled to undergo SLNB participated in this study. Both Sonazoid ${ }^{\circledR}$ and dye were used as tracers, and the most upstream sentinel lymph node (SLN) at each lymphatic flow detected by CEUS (First-SLN) was sampled under hookwire guidance, a procedure called "Sona-Hook". Results: In each of the 50 cases, at least one First-SLN was extracted by "Sona-Hook". All contrast-enhanced SLNs (CE-SLNS) were dye-positive, and the mean number of CE-SLNs sampled per patient was lower than that of dye-positive SLNs (1.48 vs. 1.88, $p<0.01)$. Through OSNA, qualitative assessment of tumor metastasis between First-SLNs and all SLNs completely matched together. Conclusion: "Sona-Hook" for First-SLN followed by an OSNA assay may be a feasible minimally invasive SLNB strategy.
\end{abstract}

Sentinel lymph node biopsy (SLNB) for clinically nodenegative breast cancer has been established as a standard minimally invasive procedure for axillary staging. Sentinel lymph nodes (SLNs) are conventionally identified using a radioisotope (RI) and blue dye. The RI method is easy to perform and helps surgeons identify SLNs with certainty; however, there are related issues, such as a limited number

This article is freely accessible online.

Correspondence to: Kenzo Shimazu, MD, Department of Breast and Endocrine Surgery, Osaka University Graduate School of Medicine, 2-2-E10 Yamada-oka, Suita City, Osaka 565-0871, Japan. Tel: +81 0668793772, Fax: +81 0668793779, e-mail: kshimazu@onsurg.med.osaka-u.ac.jp

Key Words: Breast cancer, sentinel lymph node biopsy, one-step nucleic acid amplification (OSNA), hookwire, contrast-enhanced ultrasonography (CEUS), Sona-Hook. of facilities that can handle RIs as well as radiation exposure. On the other hand, although the blue dye method alone does not necessitate a special facility for radiation protection, time is required to master the procedure, and missing any of the lymphatic flows produces a false-negative result when multiple lymphatic flows are present. Actually, it has been reported that the false negative rate of SLNB is higher in the blue dye method alone than in the combination method using both blue dye and an RI (1).

To potentially compensate for this weakness of the dye method alone, a near-infrared fluorescence system using green dye (indocyanine green: ICG) to detect SLNs has been reported to be useful $(2,3)$. However, there are problems such as the necessity of expensive fluorescent infrared cameras and difficulty in observing detailed lymph flow with fluorescent cameras when the lymph vessels are damaged and ICG diffuses to the surroundings. Considering that $33 \%$ of hospitals in Japan adopt the blue dye method alone for SLNB (4), the development of an alternative concise and accurate SLNB method is needed.

Recently, SLNB using contrast-enhanced ultrasound (CEUS) has been reported (5-10). Peri-areolar injection of an ultrasound contrast agent visualized the lymphatic flow from breast to axilla, and the identification rate of SLN by CEUS (CE-SLN) was between $70 \%$ and $100 \%$. A standard surgical technique for SLNB using CEUS has not been established. In these studies, ultrasound contrast agent was used for SLNB in combination with blue dye and an RI $(5,7,8)$, blue dye and computed tomographic (CT) lymphography (9), blue dye alone $(6,10)$, blue dye and ICG (6), and ICG alone (6). For the purpose of sampling CE-SLNs, their localization was noted by marking the skin with ink $(5,6,9)$. As a more precise CE-SLN localization method, ultrasonography-guided placement of a hookwire in CE-SLN has been successfully developed $(7,8,10)$.

Pathological diagnosis has been a conventional, standard method for the diagnosis of metastasis in SLNs. In recent years, a one-step nucleic acid amplification (OSNA) 
diagnostic assay (Sysmex Corporation, Kobe, Japan) for SLN metastasis has been developed that enables us to examine whole SLNs in a short time during operations, with a high sensitivity (11-13). Because metastasis in CE-SLN has been diagnosed by means of pathological diagnosis in previous studies, confirmation of the utility of the OSNA diagnostic assay for CE-SLN will broaden the performance of SLNB by the CEUS method in hospitals that adopt the OSNA assay for SLNB.

In the present study, we investigated the utility of hookwire-guided SLNB using an ultrasound contrast agent (perflubutane microbubble: Sonazoid ${ }^{\circledR}$; Daiichi-Sankyo, Tokyo, Japan) and dye, named "Sona-Hook", to accurately sample the most upstream SLN (First-SLN) at each lymphatic flow. In addition, we examined the feasibility of the OSNA diagnostic assay for CE-SLN. With regard to the dye used as a tracer for SLNB, the ICG fluorescence system (which has not been used in combination with Sonazoid ${ }^{\circledR}$ in previous studies except for our study) (6) or patent blue were administered to each patient.

\section{Patients and Methods}

Patients. Clinically T1-2N0M0 breast cancer patients $(\mathrm{n}=52)$ who had been scheduled to undergo SLNB at Osaka University Hospital between November 2017 and October 2018 were recruited in this study. Male breast cancer patients and those treated with neoadjuvant therapy were excluded. Two patients withdrew from our study the day before the operation; thus, a total of 50 patients took part in the study. The patients' clinical and pathological characteristics are shown in Table I. The cut-off value for the Ki67 labeling index was determined to be $20 \%$. Surgical procedures such as total mastectomy or partial mastectomy were selected according to multiple parameters, including tumor location, breast size, patient preference, and so on. Immediate breast reconstruction was performed in 29\% (9 of 31) of the patients undergoing total mastectomy. This prospective study was approved by the institutional review board of Osaka University Hospital (approval number 17126), and informed consent was obtained from each patient before surgery.

Sentinel lymph node biopsy. SLNB was performed using an ultrasound contrast agent (perflubutane microbubble: Sonazoid ${ }^{\circledR}$; Daiichi-Sankyo, Tokyo, Japan) in combination with a dye (patent blue: $n=40$; ICG: $n=10$ ), and metastasis in SLN was detected by means of an OSNA assay (Sysmex Corporation, Kobe, Japan) (6, 12, 13). After periareolar injection of patent blue or ICG in an operating theater, Sonazoid ${ }^{\circledR}$ was injected into the adjacent area of the dye and manually massaged for 1-2 minutes. Then, lymphatic flow from the breast to the axilla was identified by contrastenhanced ultrasound (CEUS) using Aplio i $700^{\circledR}$ (Canon Medical Systems, Otawara, Japan) under the same conditions as Aplio $500^{\circledR}$ (6). The mechanical index and frame rate were set to 0.2 and 13 frames per second, respectively. After a short skin incision was made, a hookwire (D Wire BLN ${ }^{\circledR}$, Argon Medical Devices, Inc., Athens, TX, USA) was indwelled under ultrasonic guidance to the most upstream CE-SLN (First-SLN) at each lymphatic flow, and the
Table I. Clinical and pathological characteristics of breast cancer patients.

\begin{tabular}{lc}
\hline & Number of patients $(\%)$ \\
\hline Menopausal status & \\
Pre & $17(34)$ \\
Post & $33(66)$ \\
Clinical tumor size & $39(78)$ \\
T1 & $11(22)$ \\
T2 & \\
Histology & $38(76)$ \\
Ductal & $2(4)$ \\
Lobular & $3(6)$ \\
Mucinous & $7(14)$ \\
Others & $16(32)$ \\
Histological grade & $24(48)$ \\
I & $10(20)$ \\
II & \\
III & $41(82)$ \\
Estrogen receptor & $9(18)$ \\
Positive & \\
Negative & $32(64)$ \\
Progesterone receptor & $18(36)$ \\
Positive & $10(20)$ \\
Negative & $40(80)$ \\
HER2 & $30(60)$ \\
Positive & $20(40)$ \\
Negative & $31(62)$ \\
Ki67 & $19(38)$ \\
Positive & \\
Negative & \\
Types of surgery & \\
Mastectomy & \\
Lumpectomy & \\
\hline
\end{tabular}

Figure 1. Procedure of hookwire-guided sentinel lymph node biopsy using a contrast agent and a blue dye, a protocol called "Sona-Hook". The "Sona-Hook" procedure is presented. After periareolar injection of Sonazoid ${ }^{\circledR}(A)$, lymphatic flow from breast to axilla was observed by CEUS (B). The parallel display of CEUS shows a contrast-enhanced $S L N$ located in the most upstream portion of a lymphatic flow (First$S L N)$ in a CEUS-mode setting (C, left) but not in a normal B-mode setting $(C$, right). After a short skin incision was made, the hookwire was inserted into the axilla $(D)$ and was confirmed to be correctly positioned in the First-SLN (white arrow, E). Following placement of the wire, the insertion needle covering the wire was removed (yellow arrow, F). Subsequently, the skin incision was extended, the subdermal axillary tissues surrounding the hookwire were dissected while pulling the wire, and the First-SLN dyed with patent blue was detected $(G)$ and then extracted $(H)$. CEUS confirmed that Sonazoid ${ }^{\circledR}$ remained inside the sampled First-SLN (I), and there were no remaining CE-SLNs in the axilla $(J)$. CEUS, contrast-enhanced ultrasonography; SLN, sentinel lymph node; CE-SLN, contrast-enhanced sentinel lymph node. 

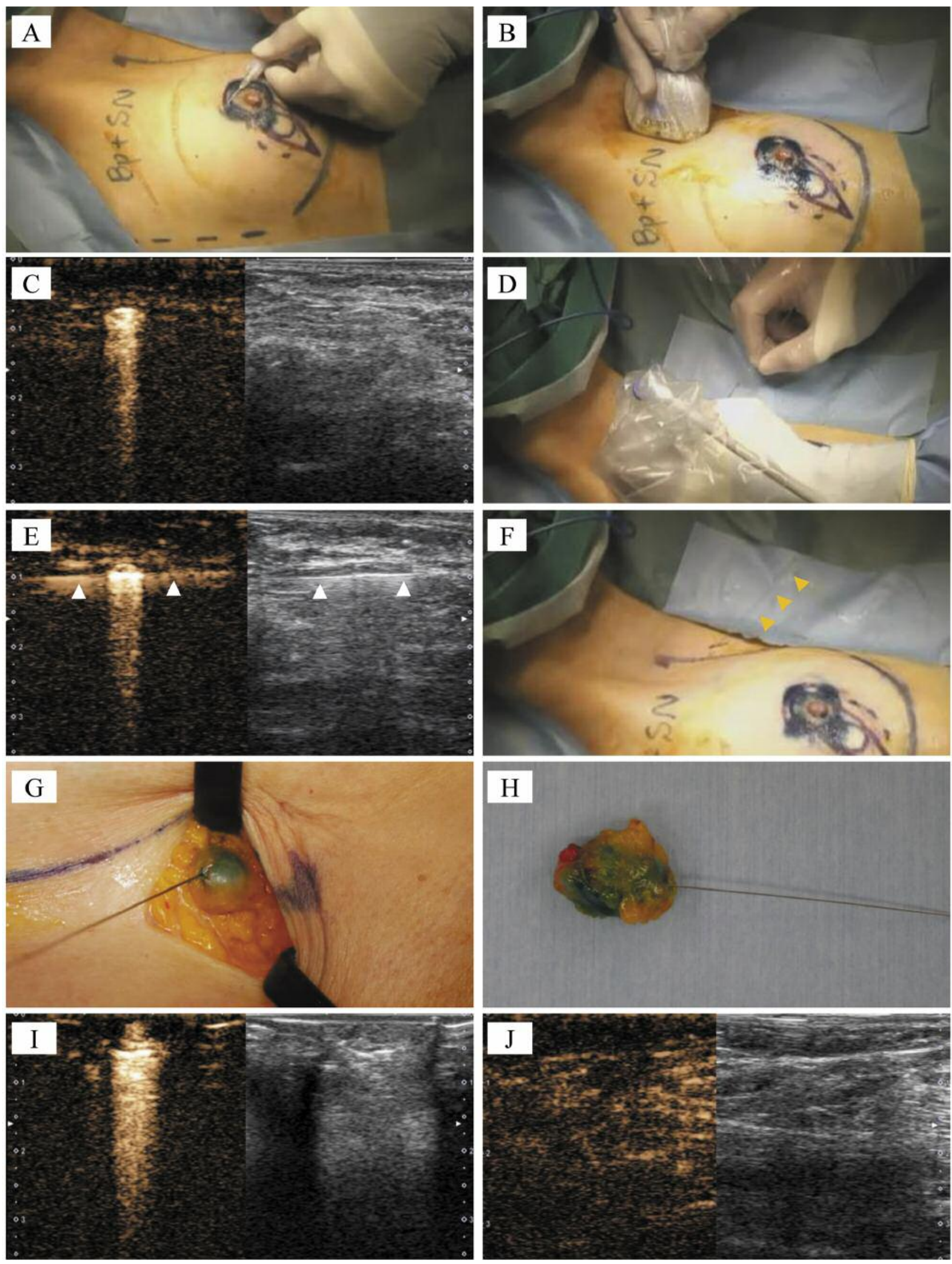
Table II. In vitro feasibility study of sentinel lymph node biopsy using contrast agent by means of one step nucleic acid amplification system (n=2).

\begin{tabular}{|c|c|c|c|c|c|c|}
\hline \multirow{2}{*}{$\begin{array}{l}\text { Concentration of } \\
\text { Sonazoid }^{\circledR}(\%)\end{array}$} & \multicolumn{2}{|c|}{ CK19 mRNA low sample $(+)$} & \multicolumn{2}{|c|}{ CK19 mRNA high sample $(++)$} & \multicolumn{2}{|c|}{ CK19 mRNA negative sample (-) } \\
\hline & $\begin{array}{c}\text { CK19 mRNA } \\
\text { (mean of copies/ } / \mu 1)\end{array}$ & $\begin{array}{l}\text { Qualitative } \\
\text { assessment }\end{array}$ & $\begin{array}{c}\text { CK19 mRNA } \\
\text { (mean of copies } / \mu 1)\end{array}$ & $\begin{array}{l}\text { Qualitative } \\
\text { assessment }\end{array}$ & $\begin{array}{c}\text { CK19 mRNA } \\
\text { (mean of copies } / \mu 1)\end{array}$ & $\begin{array}{l}\text { Qualitative } \\
\text { assessmen }\end{array}$ \\
\hline 0.04 & $1.7 \times 10^{3}$ & $(+)$ & $2.4 \times 10^{5}$ & $(++)$ & ND & $(-)$ \\
\hline 0.004 & $2.7 \times 10^{3}$ & $(+)$ & $2.9 \times 10^{5}$ & $(++)$ & ND & $(-)$ \\
\hline 0.0004 & $4.1 \times 10^{3}$ & $(+)$ & $2.9 \times 10^{5}$ & $(++)$ & ND & $(-)$ \\
\hline 0 & $2.7 \times 10^{3}$ & $(+)$ & $2.9 \times 10^{5}$ & $(++)$ & ND & $(-)$ \\
\hline
\end{tabular}

++: Macrometastasis; +: Micrometastasis; -: Negative.

First-SLN was sampled while pulling the wire (called "SonaHook"). Downstream CE-SLNs, identified preoperatively, were also sampled by the "Sona-Hook" procedure. Following the First-SLN sampling, dye-positive SLNs were additionally biopsied. All extracted SLNs were examined by CEUS to investigate the remaining contrast agent inside them. Moreover, the axilla was subsequently examined by CEUS to confirm that all CE-SLNs had been extracted. When ICG was used for SLNB, ICG in the lymphatic flow from breast to axilla and SLNs were confirmed using a near-infrared monitor (Hyper Eye Medical System ${ }^{\circledR}$, Mizuho Medical Co., Ltd., Tokyo, Japan). A representative "Sona-Hook" procedure is illustrated in Figure 1.

At the beginning of the study, the accuracy of the OSNA diagnostic assay for SLNs containing Sonazoid ${ }^{\circledR}$ was examined in vitro $(\mathrm{n}=2)$. In the OSNA assay, SLNs are homogenized by $4 \mathrm{ml}$ of lysis buffer, and each sample is diluted ten times. Therefore, assuming that a total amount of $16 \mu \mathrm{l}\left(=1\right.$ vial) of Sonazoid ${ }^{\circledR}$ reached an SLN, the final added concentration of Sonazoid ${ }^{\circledR}$ in the sample tube was $0.04 \%(\mathrm{v} / \mathrm{v})$, which was defined as the top concentration. Then, $0.04 \%, 0.004 \%, 0.0004 \%$ and $0 \%$ of Sonazoid ${ }^{\circledR}$ were added to OSNA lysis buffer in each sample tube, respectively, and the OSNA assay was conducted for the CK19 mRNA high sample, CK19 mRNA low sample and CK19 mRNA negative sample, respectively (Table II). Because the qualitative assessment did not differ irrespective of the concentration of Sonazoid ${ }^{\circledR}$, we concluded that Sonazoid ${ }^{\circledR}$ did not affect the results of the OSNA assay in vitro.

Statistical analysis. The number of SLNs sampled per patient according to the dye and/or Sonazoid ${ }^{\circledR}$ was compared using a paired $t$-test. JMP ${ }^{\circledR}$ PRO 14 software (SAS Institute, Inc., Cary, NC, USA) was used for all statistical analyses. $p$-Values $<0.05$ were determined to indicate statistically significant differences.

\section{Results}

"Sona-Hook" for First-SLNs. In all cases, lymphatic flow from breast to axilla was clearly visualized, and at least one First-SLN was detected by CEUS. Details of the lymphatic flow to First-SLNs were as follows: one lymph duct flowed into one First-SLN (n=33), two lymph ducts flowed into one First-SLN (n=2), and two lymph ducts flowed into two FirstSLNs separately $(n=15)$. The median size of 65 First-SLNs in 50 cases was $8.4 \mathrm{~mm}$ (range=3.5-19.3 mm). All First-SLNs were sampled successfully under hookwire guidance, and it was confirmed that the hookwire was positioned inside $(n=47)$ or adjacent to the surrounding tissue $(n=18)$ of the First-SLNs. Additionally, the remaining contrast agent inside all extracted First-SLNs was confirmed by CEUS. A small hematoma with a diameter of approximately $2 \mathrm{~cm}$ due to the hookwire insertion was observed in the axilla of one case, but no severe complications were observed in the present study.

Association of the number of sampled SLNs according to different tracers for $S L N B$. Based on the analysis on a nodeby-node basis, 68 CE-SLNs, including 65 First-SLNs and 3 downstream CE-SLNs, were identified preoperatively and were sampled by "Sona-Hook". In addition, 5 downstream SLNs containing both dye and Sonazoid ${ }^{\circledR}$ were detected by trimming of sampled First-SLNs, and 3 SLNs sampled by the dye method were shown to contain Sonazoid ${ }^{\circledR}$. As a result, 76 SLNs containing Sonazoid ${ }^{\circledR}$ were sampled and confirmed to be dye-positive. On the other hand, the number of SLNs detected by dye alone was 18 . Thus, the total number of extracted SLNs was 94. Based on the analysis on a patientby-patient basis, only First-SLNs were detected as CE-SLNs in $94 \%$ (47 of 50) of the patients and were sampled by "SonaHook". As a result, the mean number of SLNs sampled by "Sona-Hook" for each patient was lower than that sampled by the dye procedure $(1.48 v s .1 .88, p<0.01)$.

Association of metastasis diagnosis between only First-SLNs and all SLNs. In the present study, 4 cases (8\%) and 5 cases $(10 \%)$ were shown to have macrometastasis and micrometastasis in SLNs, respectively. Detailed metastatic information of the SLNs in these 9 cases is shown in Figure 2. Among the 9 SLN metastatic cases, 4 cases with SLN macrometastasis and 1 case with SLN micrometastasis underwent additional axillary lymph node dissection (ALND), and 3 of 4 cases with macrometastasis had nonSLN metastasis. In each metastatic case, at least one FirstSLN harbored metastasis. On the other hand, of 41 cases 
Case 1

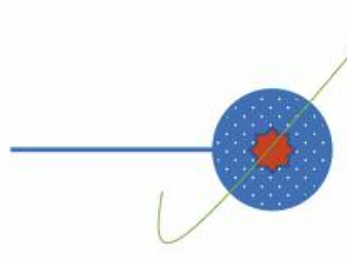

Case 5

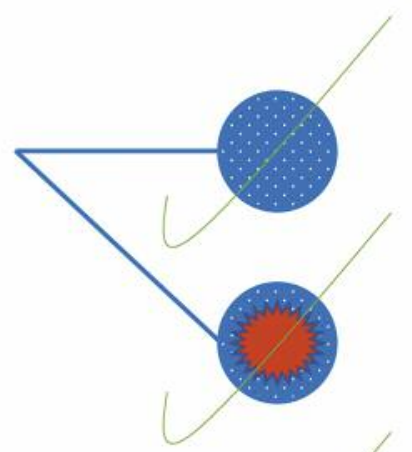

Case 18

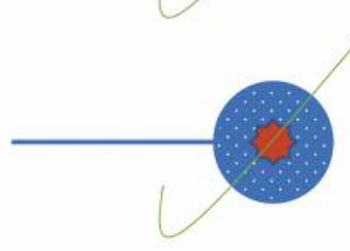

Case 22

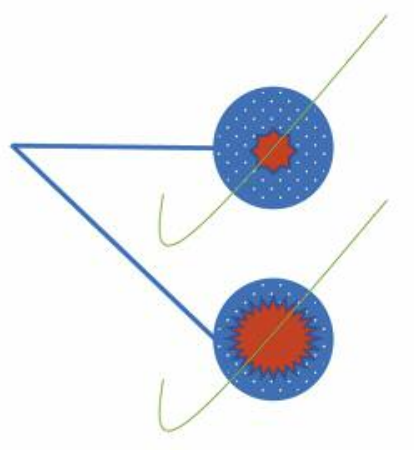

Case 36

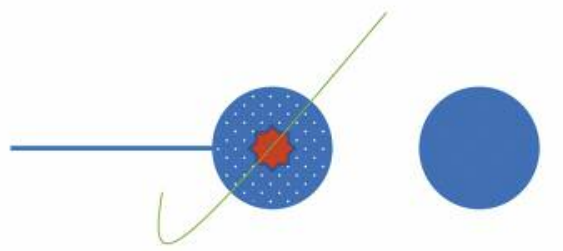

Case 2

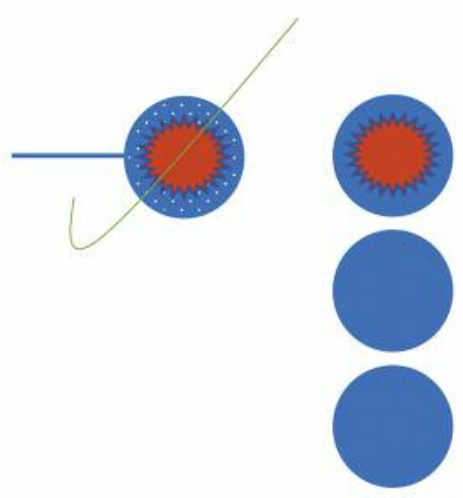

Case 10
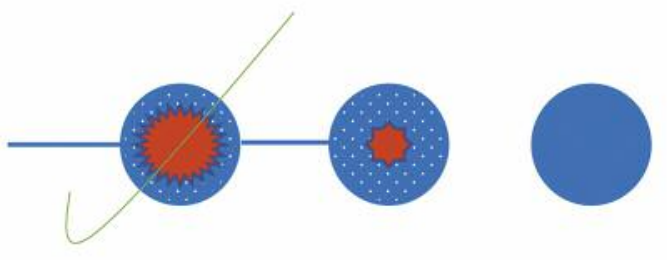

Case 19
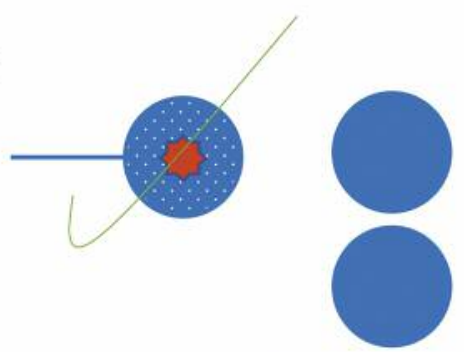

Case 33
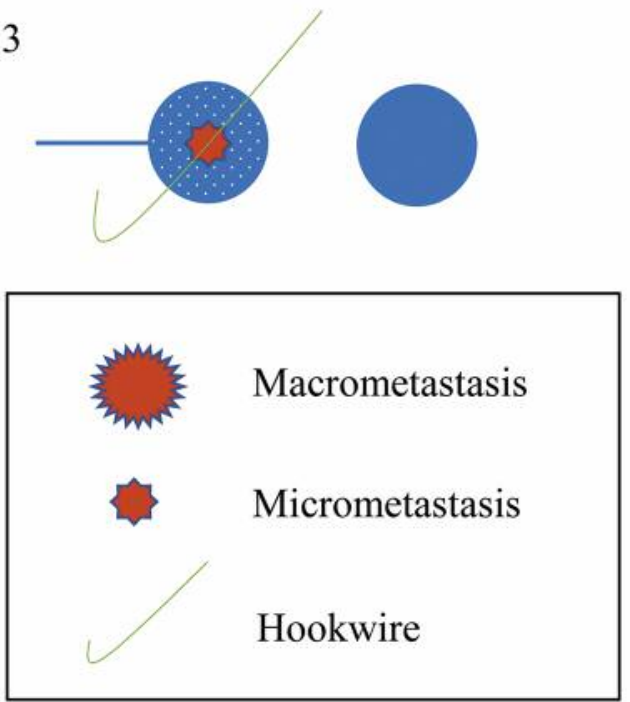

Figure 2. Case series of breast cancer patients whose sentinel nodes harbored metastasis. Detailed axillary status of nine cases with SLN metastasis, including macrometastasis in 4 cases and micrometastasis in 5 cases, are presented. All First-SLNs, which are defined as the most upstream SLNs of lymphatic flows (blue lines) from breast to axilla, were positive for both ultrasound contrast agent and dye (blue circle with dots inside). SLNs that were positive for dye alone (blue circle without dots) were identified in 5 cases. Metastatic diagnosis based on the findings of First-SLNs sampled by the "Sona-Hook" procedure reflected the diagnosis based on whole SLNs. SLN, sentinel lymph node. 

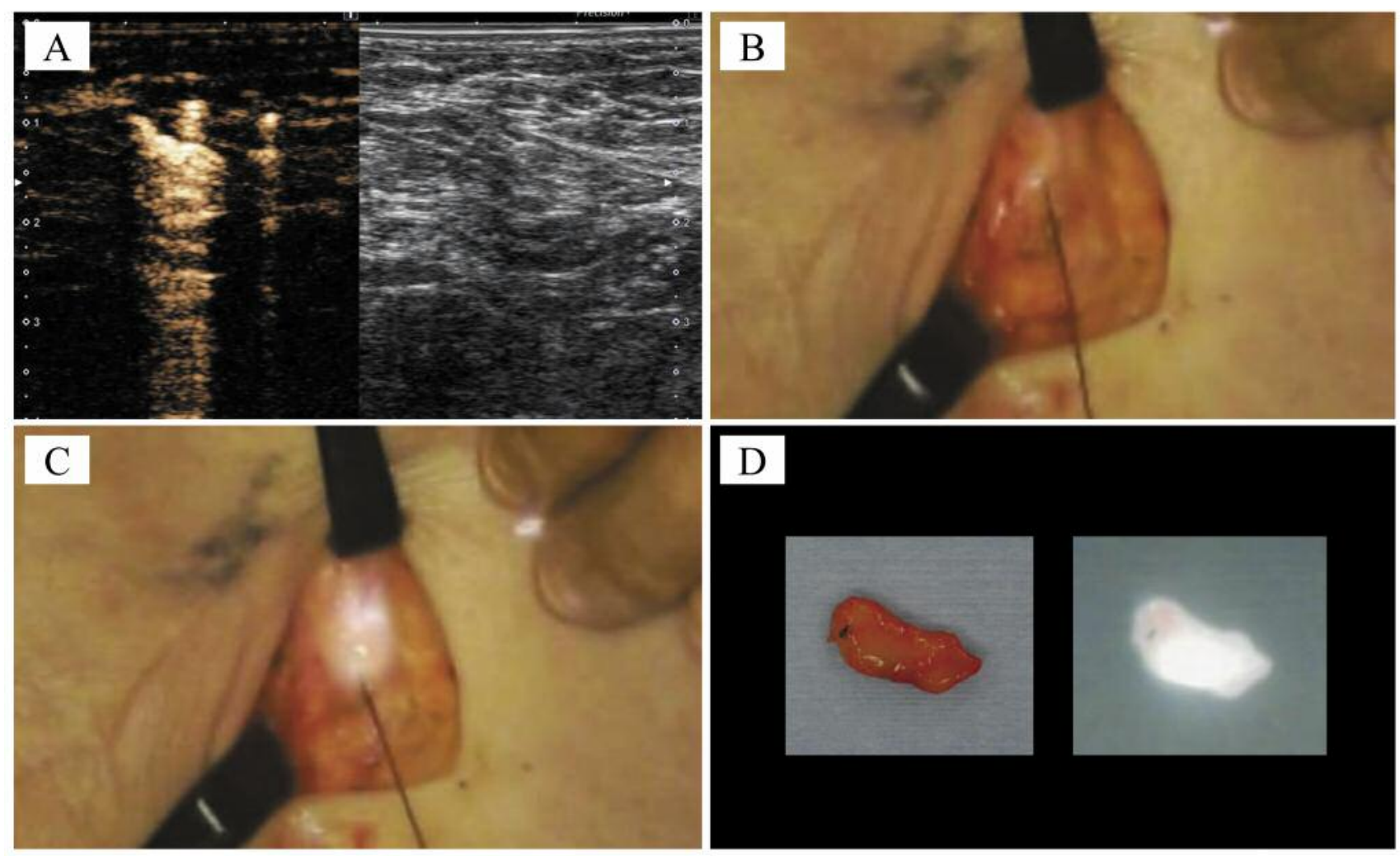

Figure 3. Representative procedure of sentinel node biopsy using a contrast agent and green dye (ICG). SLNB using Sonazoid ${ }^{\circledR}$ in combination with ICG was performed on 10 patients. A CE-SLN was detected in the axilla (A), and subsequently, a hookwire was placed in the SLN according to the "Sona-Hook" procedure. At the beginning of dissection of the axillary subdermal tissue surrounding the wire, ICG in the SLN was not visualized by a near-infrared camera $(B)$ but was clearly visualized by the camera by pulling the hookwire $(C)$. An extracted CE-SLN stained by ICG was unclear to the naked eye $(D$, left), whereas ICG inside the SLN was clearly identified by the near-infrared camera $(D$, right). SLNB, Sentinel lymph node biopsy; ICG, indocyanine green; CE-SLN, contrast-enhanced sentinel lymph node.

without First-SLN metastasis, 18 cases had additional downstream SLNs, and none of them harbored metastasis. The association of the metastatic diagnosis between FirstSLN and whole SLNs is shown in Table III. Consequently, diagnosis based on the findings of First-SLNs and that based on the findings of all SLNs, which also include SLNs detected by dye alone, completely matched each other.

\section{Discussion}

In the present study, we investigated the feasibility of the "Sona-Hook" procedure. First, lymphatic flow from the breast to axilla was clearly visualized by periareolar injection of Sonazoid ${ }^{\circledR}$ using CEUS, and First-SLNs were identified in all cases, yielding an identification rate that was almost the same as that reported in previous studies $(6,9)$. Subsequently, all First-SLNs were successfully sampled under hookwire guidance, which was also the same result as in previous reports $(7,8,10)$. For 10 of 50 patients,
Table III. Association of metastatic diagnosis between First-SLNs and all SLNS.

Final diagnosis based on all SLNs per patient $(\mathrm{n}=50)$

Macrometastasis Micrometastasis Negative

\begin{tabular}{llrl}
\hline Diagnosis based on & & & \\
First-SLNs & 4 & 0 & 0 \\
Macrometastasis & 0 & 5 & 0 \\
Micrometastasis & 0 & 0 & 41 \\
Negative & & \\
\hline
\end{tabular}

SLN, Sentinel lymph node.

lymphatic flow and SLNs were visualized by periareolar injection of ICG using a near-infrared camera at the same time as CEUS, and First-SLNs were clearly demonstrated to be ICG-positive (Figure 3). As we have reported previously, no severe complications were observed in the study (6). 


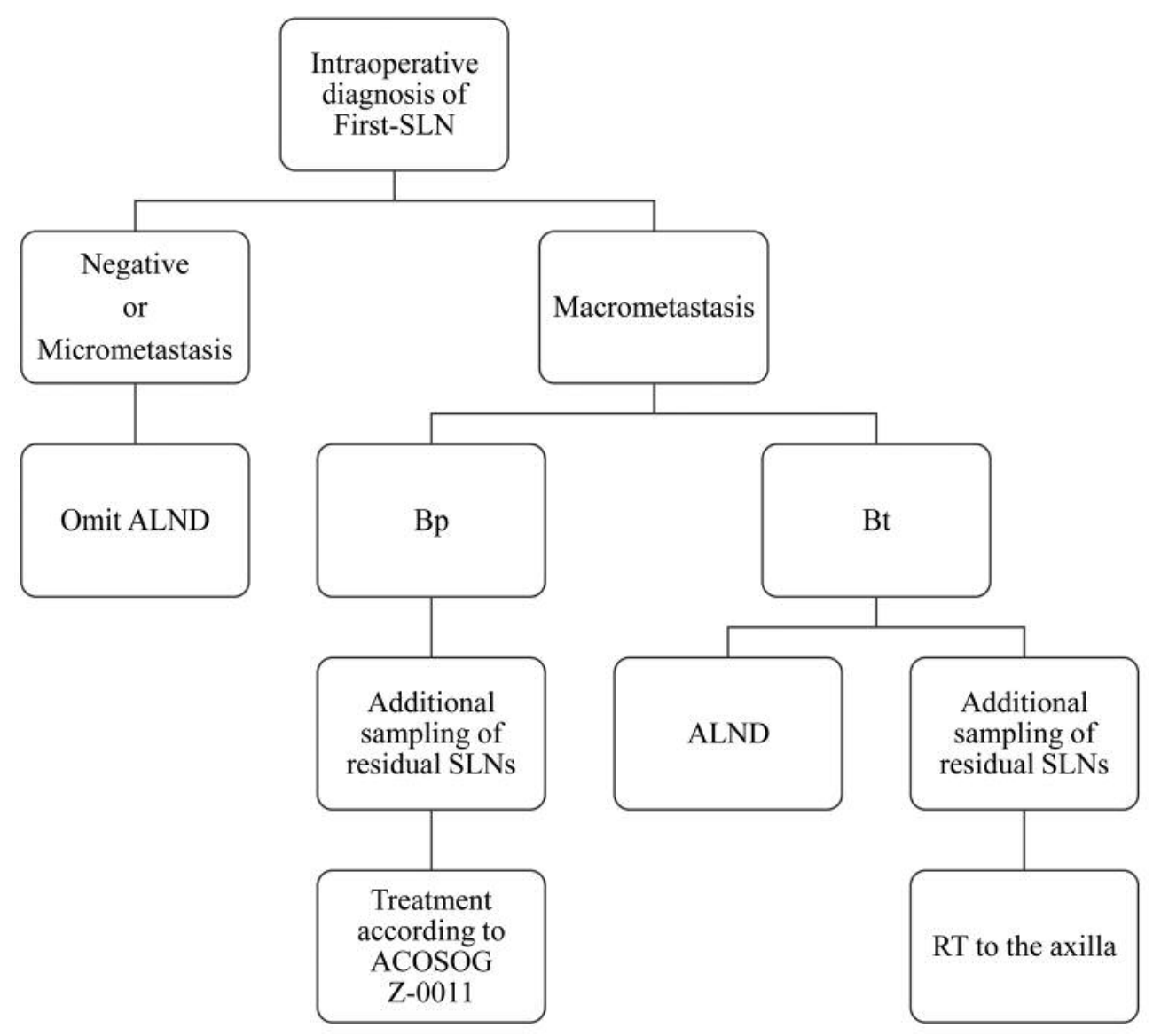

Figure 4. Flow chart of treatment based on intraoperative diagnosis of First-SLNs sampled by the "Sona-Hook" procedure. A proposal of the decision tree for the management of SLNB is shown. First, "Sona-Hook" followed by an intraoperative detection method of SLN metastasis such as OSNA or conventional pathological assay is recommended as an initial SLNB for First-SLNs. Next, if there is no metastasis or only micrometastasis in the First-SLNs, ALND can be spared. In case macrometastasis is detected in First-SLNs of a patient undergoing breast-conserving surgery, additional sampling of residual SLNs in the axilla followed by treatment according to the ACOSOG-Z0011 criteria is recommended. On the other hand, when macrometastasis is found in the First-SLNs of patients undergoing breast mastectomy, conventional ALND or additional sampling of residual SLNs followed by radiation therapy to the axilla as an option are recommended. SLNB, Sentinel lymph node biopsy; SLN, sentinel lymph node; OSNA, one-step nucleic acid amplification; ALND, axillary lymph node dissection; Bp, breast-conserving surgery; Bt, breast mastectomy; ACOSOG, American College of Surgeons Oncology Group; RT, radiation therapy.

Next, differences in the number of SLNs sampled according to the SLN identification method were analyzed. As a result, a patient-by-patient analysis demonstrated that the mean number of CE-SLNs was significantly lower than that of dye-positive SLNs and that all CE-SLNs were dyepositive. This result is the same as that in our previous reports (6), suggesting that Sonazoid ${ }^{\circledR}$ tends to remain in the upstream SLNs longer than the dye, possibly because the mean diameter of Sonazoid ${ }^{\circledR}(2-3 \mu \mathrm{m})$ is larger than that of the dye. In fact, $96 \%$ (65 in 68) of CE-SLNs identified preoperatively were First-SLNs, and only $4 \%$ of them were downstream SLNs. Consequently, the application of Sonazoid ${ }^{\circledR}$ as a tracer for SLNB may enable us to perform a more efficient SLNB.
In our study, $18 \%$ of the total patients showed macrometastasis $(8 \%)$ or micrometastasis $(10 \%)$ in SLNs. The patient-by-patient analysis showed that the diagnosis based on the results of First-SLNs was perfectly consistent with that based on the results of whole SLNs. This result led us to re-realize the significance of the sampling of FirstSLNs for an accurate SLNB. Considering our results showing that the mean number of SLNs sampled by the "Sona-Hook" procedure was lower than that sampled by the dye procedure and that the diagnoses based on the First-SLN findings completely aligned with those based on the findings for whole SLNs, sampling only First-SLNs is expected to enable us to minimize unnecessary surgical manipulation of the axilla, leading to a minimally invasive SLNB. 
Based on the analysis of lymphatic flow from the breast to axilla, $30 \%$ of the patients were shown to have two lymphatic flows to two SLNs, and in one of these patients (case 5), metastasis was found in one SLN. In such a case, SLNB using the dye-alone method poses a risk for overlooking one of the lymphatic flows to a First-SLN, producing a false-negative result. SLNB using the dye-only method has been reported to yield a lower identification rate as well as a higher false-negative rate than those using both dye and RI $(1,14)$. On the other hand, SLNB using both dye and an ultrasound contrast agent yielded a high identification rate in our present and past trials, although the false-negative rate has not been investigated (6). Therefore, the combination method using a dye and an ultrasound contrast agent for SLNB may contribute to reducing the risk of overlooking First-SLNs, leading to more precise axillary staging.

The OSNA assay, but not the conventional pathological assay, was used for the detection of SLN metastasis in the present study. This assay enables us to determine the necessity of additional ALND during the operation for patients undergoing total mastectomy or those undergoing partial mastectomy and do not meet the criteria of the American College of Surgeons Oncology Group (ACOSOG) Z-0011 (15). In addition, we developed a nomogram to predict four or more axillary lymph node metastases based on the OSNA results (16). Therefore, the OSNA assay in combination with the nomogram also facilitates the decision regarding optimal adjuvant therapy for patients undergoing breast partial mastectomy and meet the criteria of ACOSOG Z-0011. At the beginning of this study, it was confirmed that the use of Sonazoid ${ }^{\circledR}$ does not affect the results of the OSNA diagnostic assay in vitro. As an in vivo finding, $18 \%$ of patients showed macro- or micro-metastasis in SLNs, which is a slightly lower frequency than that reported in the previous reports $(17,18)$. This may be because $78 \%$ of the patients participated in this study had clinical stage I tumors that had a low possibility of disseminating to the axilla. On the other hand, as mentioned above, the diagnosis of SLN metastasis was completely in accordance between First-SLNs and all SLNs that also contained SLNs detected by dye alone, demonstrating that the ability of OSNA to diagnose CE-SLNs is the same as that for SLNs that contain dye alone.

In recent years, the combination of microbubble technology and ultrasound has shed light on the field of antitumor therapy, such as drug delivery systems (19-21) and hyperthermia (22), as well as the diagnosis of cancer (23). A drug delivery system using microbubbles and US is reportedly based on the finding that microjets produced by oscillations or destruction of the microbubbles increase the permeability of vessel membranes $(24,25)$. On the other hand, the use of hyperthermia is reported to be based on the fact that destruction of microbubbles induced by acoustic exposure increases the temperature around a tumor (22). In the present study, perflubutane microbubbles were used as a tracer for SLNB, and SLNs were detected by US. Perflubutane tends to be destroyed under the conditions of a high mechanical index (MI) and a high frame rate, and the possibility that microbubbles under the conditions of our study damage the surrounding tissue is low (26). In fact, CEUS-guided liver needle biopsy using perflubutane microbubbles under conditions similar to our study has been reported to be useful (27). In addition, in this study, we could not find any specific abnormal alteration of the cellular morphology or protein expression of CE-SLNs by means of hematoxylin-eosin or immuno-histological staining compared to the findings for SLNs detected by dye alone (data not shown). In summary, performing SLNB by the "Sona-Hook" procedure followed by the OSNA and pathological diagnostic assays seems clinically reasonable.

Based on the results obtained from the present study, a decision tree for the management of SLNB can be proposed, although future studies including larger patient samples are needed to evaluate this strategy (Figure 4). First, "SonaHook" followed by intraoperative detection of SLN metastasis using OSNA or conventional pathological assay is recommended as an initial SLNB for First-SLN. Next, if there is no metastasis or only micrometastasis in the FirstSLNs, ALND can be spared. If macrometastasis is detected in First-SLNs of a patient undergoing breast-conserving surgery, then additional sampling of residual SLNs in the axilla followed by treatment according to the ACOSOG Z0011 criteria, which indicate the possibility of omission of ALND for clinical T1-2 breast cancer patients with at most two SLN macrometastases who will be treated with optimal systemic therapy, is recommended (15). On the other hand, when macrometastasis is found in the First-SLNs of patients undergoing breast mastectomy, conventional ALND or additional sampling of residual SLNs followed by radiation therapy to the axilla as an option is recommended $(28,29)$. In the application of the decision tree using the "Sona-Hook" procedure to the patients of the present study, $92 \%$ (46 in 50) of the patients could be treated with only First-SLN biopsy for axillary staging; thus, it is expected that the decision tree will give such patients the benefit of undergoing minimally invasive axillary staging. In addition, OSNA can provide us with the metastatic information on SLNs intraoperatively based on the examination of the whole specimen; thus, OSNA has the advantage that it does not contain a risk of a mismatch between intra- and postoperative diagnosis, whereas conventional intraoperative pathological diagnosis has a risk of a false negative result. Furthermore, an intraoperative predictive model for non-SLN metastasis based on the finding of SLN metastasis by means of OSNA has been developed recently (16) and is expected to help us choose the indication for additional ALND. 
In conclusion, our results indicated that a hookwire-guided First-SLN biopsy using Sonazoid ${ }^{\circledR}$, named "Sona-Hook", was successfully performed and shown to be technically feasible for SLNB. In addition, the diagnostic feasibility of the OSNA assay for SLNs containing Sonazoid ${ }^{\circledR}$ was demonstrated. First-SLN biopsy by means of "Sona-Hook" followed by OSNA may enable us to perform accurate and minimally invasive SLNB. The results of our study need to be validated in a larger prospective study.

\section{Conflicts of Interest}

Dr. Noguchi has received research funding and honoraria from Daiichi-Sankyo Co., Ltd., and Sysmex Corporation for work performed outside of the current study. Dr. Shimazu received honoraria from Sysmex Corporation for work performed outside of the current study. Dr. Naoi received research funding and honoraria from Sysmex Corporation for work performed outside of the current study. This research did not receive any specific grant from funding agencies in the public, commercial, or not-for-profit sectors.

\section{Authors' Contributions}

TM, KS and SN designed the study, and wrote the initial draft of the manuscript. All Authors have contributed to data collection, analysis and interpretation, and critically reviewed the manuscript. All Authors approved the final version of the manuscript, and agree to be accountable for all aspects of the work in ensuring that questions related to the accuracy or integrity of any part of the work are appropriately investigated and resolved.

\section{Acknowledgements}

The Authors are grateful to Hiroki Shoji of the Laboratory for Clinical Investigation at Osaka University Hospital for performing the OSNA diagnostic assay.

\section{References}

1 Noguchi M, Motomura K, Imoto S, Miyauchi M, Sato K, Iwata $\mathrm{H}$, Ohta $\mathrm{M}$, Kurosumi $\mathrm{M}$ and Tsugawa $\mathrm{K}$ : A multicenter validation study of sentinel lymph node biopsy by the japanese breast cancer society. Breast Cancer Res Treat 63(1): 31-40, 2000. PMID: 11079157. DOI: 10.1023/a:1006428105579

2 Sugie T, Sawada T, Tagaya N, Kinoshita T, Yamagami K, Suwa $\mathrm{H}$, Ikeda T, Yoshimura K, Niimi M, Shimizu A and Toi M: Comparison of the indocyanine green fluorescence and blue dye methods in detection of sentinel lymph nodes in early-stage breast cancer. Ann Surg Oncol 20(7): 2213-2218, 2013. PMID: 23429938. DOI: 10.1245/s10434-013-2890-0

3 Verbeek FP, Troyan SL, Mieog JS, Liefers GJ, Moffitt LA, Rosenberg M, Hirshfield-Bartek J, Gioux S, van de Velde CJ, Vahrmeijer AL and Frangioni JV: Near-infrared fluorescence sentinel lymph node mapping in breast cancer: A multicenter experience. Breast Cancer Res Treat 143(2): 333-342, 2014. PMID: 24337507. DOI: 10.1007/s10549-013-2802-9

4 Ikeda T, Sugie T, Shimizu A and Toi M: Patterns of clinical practice for sentinel lymph node biopsy in women with node- negative breast cancer: The results of a national survey in Japan. Breast Cancer 24(2): 341-344, 2017. PMID: 27568304. DOI: 10.1007/s 12282-016-0720-5

5 Omoto K, Matsunaga H, Take N, Hozumi Y, Takehara M, Omoto Y, Shiozawa M, Mizunuma H, Harashima H, Taniguchi N and Kawano M: Sentinel node detection method using contrastenhanced ultrasonography with sonazoid in breast cancer: Preliminary clinical study. Ultrasound Med Biol 35(8): 1249-1256, 2009. PMID: 19520493. DOI: 10.1016/j.ultrasmedbio.2009.02.004

6 Shimazu K, Ito T, Uji K, Miyake T, Aono T, Motomura K, Naoi Y, Shimomura A, Shimoda M, Kagara N, Kim SJ and Noguchi S: Identification of sentinel lymph nodes by contrast-enhanced ultrasonography with sonazoid in patients with breast cancer: A feasibility study in three hospitals. Cancer Med 6(8): 1915-1922, 2017. PMID: 28766883. DOI: 10.1002/cam4.1142

7 Sever AR, Mills P, Jones SE, Cox K, Weeks J, Fish D and Jones PA: Preoperative sentinel node identification with ultrasound using microbubbles in patients with breast cancer. Am J Roentgenol 196(2): 251-256, 2011. PMID: 21257873. DOI: 10.2214/AJR.10.4865

8 Esfehani MH, Yazdankhah-Kenari A, Omranipour R, Mahmoudzadeh HA, Shahriaran S, Zafarghandi MR and Amoli HA: Validation of contrast enhanced ultrasound technique to wire localization of sentinel lymph node in patients with early breast cancer. Indian J Surg Oncol 6(4): 370-373, 2015. PMID: 27065663. DOI: 10.1007/s13193-015-0446-4

9 Matsuzawa F, Omoto K, Einama T, Abe H, Suzuki T, Hamaguchi J, Kaga T, Sato M, Oomura M, Takata Y, Fujibe A, Takeda C, Tamura E, Taketomi A and Kyuno K: Accurate evaluation of axillary sentinel lymph node metastasis using contrast-enhanced ultrasonography with sonazoid in breast cancer: A preliminary clinical trial. Springerplus 4: 509, 2015. PMID: 26405629. DOI: 10.1186/s40064-015-1291-1

10 Xie F, Zhang D, Cheng L, Yu L, Yang L, Tong F, Liu H, Wang $\mathrm{S}$ and Wang S: Intradermal microbubbles and contrast-enhanced ultrasound (ceus) is a feasible approach for sentinel lymph node identification in early-stage breast cancer. World J Surg Oncol 13: 319, 2015. PMID: 26585236. DOI: 10.1186/s12957-0150736-X

11 Tamaki Y, Akiyama F, Iwase T, Kaneko T, Tsuda H, Sato K, Ueda S, Mano M, Masuda N, Takeda M, Tsujimoto M, Yoshidome K, Inaji H, Nakajima H, Komoike Y, Kataoka TR, Nakamura S, Suzuki K, Tsugawa K, Wakasa K, Okino T, Kato Y, Noguchi S and Matsuura N: Molecular detection of lymph node metastases in breast cancer patients: Results of a multicenter trial using the one-step nucleic acid amplification assay. Clin Cancer Res 15(8): 2879-2884, 2009. PMID: 19351770. DOI: 10.1158/10780432.CCR-08-1881

12 Tamaki Y, Sato N, Homma K, Takabatake D, Nishimura R, Tsujimoto M, Yoshidome K, Tsuda H, Kinoshita T, Kato H, Taniyama K, Kamio T, Nakamura S, Akiyama F, Noguchi S and Japanese One-Step Nucleic Acid Amplification Study G: Routine clinical use of the one-step nucleic acid amplification assay for detection of sentinel lymph node metastases in breast cancer patients: Results of a multicenter study in japan. Cancer 118(14): 3477-3483, 2012. PMID: 22252672. DOI: 10.1002/cncr.26683

13 Tsujimoto M, Nakabayashi K, Yoshidome K, Kaneko T, Iwase T, Akiyama F, Kato Y, Tsuda H, Ueda S, Sato K, Tamaki Y, Noguchi S, Kataoka TR, Nakajima H, Komoike Y, Inaji H, Tsugawa K, Suzuki K, Nakamura S, Daitoh M, Otomo Y and 
Matsuura N: One-step nucleic acid amplification for intraoperative detection of lymph node metastasis in breast cancer patients. Clin Cancer Res 13(16): 4807-4816, 2007. PMID: 17699859. DOI: 10.1158/1078-0432.CCR-06-2512

14 Pesek S, Ashikaga T, Krag LE and Krag D: The false-negative rate of sentinel node biopsy in patients with breast cancer: A meta-analysis. World J Surg 36(9): 2239-2251, 2012. PMID: 22569745. DOI: $10.1007 / \mathrm{s} 00268-012-1623-\mathrm{z}$

15 Giuliano AE, Ballman KV, McCall L, Beitsch PD, Brennan MB, Kelemen PR, Ollila DW, Hansen NM, Whitworth PW, Blumencranz PW, Leitch AM, Saha S, Hunt KK and Morrow M: Effect of axillary dissection vs no axillary dissection on 10-year overall survival among women with invasive breast cancer and sentinel node metastasis: The acosog z0011 (alliance) randomized clinical trial. JAMA 318(10): 918-926, 2017. PMID: 28898379. DOI: 10.1001/jama.2017.11470

16 Shimazu K, Sato N, Ogiya A, Sota Y, Yotsumoto D, Ishikawa T, Nakamura S, Kinoshita T, Tsuda H, Ohi Y, Akiyama F and Noguchi S: Intraoperative nomograms, based on one-step nucleic acid amplification, for prediction of non-sentinel node metastasis and four or more axillary node metastases in breast cancer patients with sentinel node metastasis. Ann Surg Oncol 25(9): 2603-2611, 2018. PMID: 29978372. DOI: 10.1245/s10434-018-6633-0

17 Godey F, Leveque J, Tas P, Gandon G, Poree P, Mesbah H, Lavoue V, Quillien V and Athias CB: Sentinel lymph node analysis in breast cancer: Contribution of one-step nucleic acid amplification (osna). Breast Cancer Res Treat 131(2): 509-516, 2012. PMID: 21993859. DOI: 10.1007/s10549-011-1808-4

18 Santaballa A, De La Cueva H, Salvador C, Garcia-Martinez AM, Guarin MJ, Lorente D, Palomar L, Aznar I, Dobon F and Bello P: Advantages of one step nucleic acid amplification (osna) whole node assay in sentinel lymph node $(\sin )$ analysis in breast cancer. Springerplus 2: 542, 2013. PMID: 24255842. DOI: 10.1186/2193-1801-2-542

19 Lee SK, Kim S, Choi MY, Kim J, Lee J, Jung SP, Choe JH, Kim JH, Kim JS, Kil WH, Lee JE and Nam SJ: The clinical meaning of intramammary lymph nodes. Oncology 84(1): 1-5, 2013. PMID: 23052128. DOI: 10.1159/000340016

20 Abdullgaffar B, Gopal P, Abdulrahim M, Ghazi E and Mohamed E: The significance of intramammary lymph nodes in breast cancer: A systematic review and meta-analysis. Int J Surg Pathol 20(6): 555-563, 2012. PMID: 22649167. DOI: 10.1177/106 6896912448425

21 Hogan BV, Peter MB, Shenoy H, Horgan K and Shaaban A: Intramammary lymph node metastasis predicts poorer survival in breast cancer patients. Surg Oncol 19(1): 11-16, 2010. PMID: 19171479. DOI: 10.1016/j.suronc.2008.12.009

22 Xu J, Cao Y, Xu C, Cheng X, You Y, Yao Y, Liu J, Wang Z, Li P and $\mathrm{Lu} \mathrm{M}$ : Combination of microbubbles and diagnostic ultrasound at a high mechanical index for the synergistic microwave ablation of tumours. Int J Hyperthermia 33(3): 318-326, 2017. PMID: 27764970. DOI: 10.1080/02656736.2016.1239843
23 Miyake T, Kim SJ, Shimoda M, Kagara N, Tanei T, Naoi Y, Shimazu K and Noguchi S: Diagnostic utility of third-look, contrast-enhanced sonography followed by needle biopsy for mri, but not second-look ultrasonography-detected breast lesions. Anticancer Res 39(2): 915-921, 2019. PMID: 30711976. DOI: 10.21873/anticanres.13194

24 Fan Z, Kumon RE and Deng CX: Mechanisms of microbubblefacilitated sonoporation for drug and gene delivery. Ther Deliv 5(4): 467-486, 2014. PMID: 24856171. DOI: 10.4155/tde.14.10

25 Ueno Y, Sonoda S, Suzuki R, Yokouchi M, Kawasoe Y, Tachibana K, Maruyama K, Sakamoto $T$ and Komiya S: Combination of ultrasound and bubble liposome enhance the effect of doxorubicin and inhibit murine osteosarcoma growth. Cancer Biol Ther 12(4): 270-277, 2011. PMID: 21613828. DOI: 10.4161/cbt.12.4.16259

26 Liu GJ, Moriyasu F, Hirokawa T, Rexiati M, Yamada M and Imai Y: Optical microscopic findings of the behavior of perflubutane microbubbles outside and inside kupffer cells during diagnostic ultrasound examination. Invest Radiol 43(12): 829-836, 2008. PMID: 19002054. DOI: 10.1097/RLI.0b013e3181852719

27 Lee SM, Kim JH, Yang HK, Kang HJ and Han JK: Usefulness of contrast-enhanced ultrasound using perfluorobutanecontaining microbubbles as a planning for percutaneous biopsies of focal hepatic lesions: A prospective feasibility study. Med Ultrason 21(2): 109-116, 2019. PMID: 31063512. DOI: 10.11152/mu1744

28 Donker M, van Tienhoven G, Straver ME, Meijnen P, van de Velde CJ, Mansel RE, Cataliotti L, Westenberg AH, Klinkenbijl JH, Orzalesi L, Bouma WH, van der Mijle HC, Nieuwenhuijzen GA, Veltkamp SC, Slaets L, Duez NJ, de Graaf PW, van Dalen T, Marinelli A, Rijna H, Snoj M, Bundred NJ, Merkus JW, Belkacemi Y, Petignat P, Schinagl DA, Coens C, Messina CG, Bogaerts J and Rutgers EJ: Radiotherapy or surgery of the axilla after a positive sentinel node in breast cancer (eortc 1098122023 amaros): A randomised, multicentre, open-label, phase 3 non-inferiority trial. Lancet Oncol 15(12): 1303-1310, 2014. PMID: 25439688. DOI: 10.1016/S1470-2045(14)70460-7

29 Savolt A, Peley G, Polgar C, Udvarhelyi N, Rubovszky G, Kovacs E, Gyorffy B, Kasler M and Matrai Z: Eight-year follow up result of the otoasor trial: The optimal treatment of the axilla - surgery or radiotherapy after positive sentinel lymph node biopsy in early-stage breast cancer: A randomized, single centre, phase iii, non-inferiority trial. Eur J Surg Oncol 43(4): 672-679, 2017. PMID: 28139362. DOI: 10.1016/j.ejso.2016.12.011

Received September 22, 2019 Revised October 10, 2019 Accepted October 11, 2019 